\title{
Heart-Muscle Fiber Reconstruction from Diffusion Tensor MRI
}

\author{
Leonid Zhukov \\ Alan H. Barr \\ Department of Computer Science, California Institute of Technology
}

\begin{abstract}
In this paper we use advanced tensor visualization techniques to study 3D diffusion tensor MRI data of a heart. We use scalar and tensor glyph visualization methods to investigate the data and apply a moving least squares (MLS) fiber tracing method to recover and visualize the helical structure and the orientation of the heart muscle fibers.
\end{abstract}

CR Categories: I.3.8 [Computing Methodologies]: Computer Graphics-Applications, I.6 [Computing Methodologies]: Simulation and Modeling, J.3 [Computer Application]: Life and Medical Sciences;

Keywords: Diffusion tensors, DT-MRI, fiber tracing, adaptive filtering, moving least squares, streamlines.

\section{Introduction}

Diffusion tensor magnetic resonance imaging (DT-MRI) [Basser et al. 1994] is a technique used to measure the anisotropic diffusion properties of biological tissues as a function of the spatial position within the sample. Diffusion properties allow the classification of different types of tissues and can be used for tissue segmentation and finding preferred directions in the tissue. Previously, DT-MRI data was successfully used to recover white matter fiber tracts in the brain [Basser et al. 2000; Poupon et al. 2001; Parker et al. 2001; Zhukov and Barr 2002]. Modeling of fiber orientation in the ventricular myocardium based on MR diffusion imaging was proposed by Tseng et al. [1999], Hsu and Henriquez [2001] and Sachse et al. [2001]. In this paper we apply tensor visualization and fiber reconstruction methods to DT-MRI data to recover and demonstrate the $3 \mathrm{D}$ orientation and structure of the muscle fibers in the entire heart.

In the brain, white matter fibers are detected by examining the largest eigenvalue and then "growing" the fiber along the principal eigenvector direction. This approach is effective, due to the microstructure of the white matter tissue. Heart muscle tissue has a different microstructure; as a result we have chosen to detect the fibers by examining the sum of the linear and planar tensor anisotropies (see Eq. 4), and then trace the fiber along the principal eigenvector direction as we did before.

In this paper we use a moving least squares (MLS) fiber tracing algorithm from Zhukov and Barr [2002] to recover heart muscle fibers. To our knowledge this is the first $3 \mathrm{D}$ reconstruction of the heart muscle fibers from DT-MRI data.

IEEE Visualization 2003,

October 19-24, 2003, Seattle, Washington, USA

0-7803-8120-3/03/\$17.00 @2003 IEEE

\section{Method}

\subsection{Diffusion Tensors}

Diffusion tensors describe the diffusion properties of the media, that is the ability of water molecules to move around. In biological tissues diffusion properties are dictated by the cell structure of the tissue. Water molecules can easily move inside the cell, but their motion across the cells is restricted by the cell membrane. Thus diffusion properties of the tissue reflect the shape and orientation of the cells. In the case of an elongated cell, the tissue will have a preferred diffusion direction along the primary axis of the cell.

Diffusion is measured through a diffusion coefficient, which is represented with a symmetric second order tensor - $3 \times 3$ matrix:

$$
\mathbf{D}=\left(\begin{array}{ccc}
D_{x x} & D_{x y} & D_{x z} \\
D_{y x} & D_{y y} & D_{y z} \\
D_{z x} & D_{z y} & D_{z z}
\end{array}\right)
$$

The 6 independent values (the tensor is symmetric) of the tensor elements vary continuously with the spatial location in the tissue.

Eigenvalues $\lambda_{i}$ and eigenvectors $\mathbf{e}_{i}$ of a matrix (1) can be found as a solution to the eigenvalue problem:

$$
\mathbf{D e}_{i}=\lambda_{i} \mathbf{e}_{i}
$$

Since the tensor is symmetric, its eigenvalues are always real numbers, and the eigenvectors are orthogonal and form a basis. Geometrically, a diffusion tensor can be thought of as an ellipsoid with its three axes oriented along these eigenvectors, with the three semiaxis lengths proportional to the square root of the eigenvalues of the tensor - mean diffusion distances [Basser et al. 1994].

Using the ellipsoidal interpretation, one can classify the diffusion properties of a tissue according to the shape of the ellipsoids, with extended ellipsoids corresponding to regions with strong linear diffusion (long, thin cells), flat ellipsoids to planar diffusion, and spherical ellipsoids to regions of isotropic media (such as fluidfilled regions like the ventricles). The quantitative classification can be done through the coefficients $c_{\ell}, c_{p}, c_{s}$ [Westin et al. 1997] corresponding to linear, planar and spherical diffusion.

$$
\begin{aligned}
c_{\ell} & =\frac{\lambda_{1}-\lambda_{2}}{\lambda_{1}+\lambda_{2}+\lambda_{3}} \\
c_{p} & =\frac{2\left(\lambda_{2}-\lambda_{3}\right)}{\lambda_{1}+\lambda_{2}+\lambda_{3}} \\
c_{s} & =\frac{3 \lambda_{3}}{\lambda_{1}+\lambda_{2}+\lambda_{3}}
\end{aligned}
$$

These coefficients are normalized to the range of [0..1]. Values of $c_{\ell}$ that are close to 1 selects regions with strong linear $\left(\lambda_{1}>>\right.$ $\left.\lambda_{2} \approx \lambda_{3}\right)$ diffusion. Values of $c_{p}$ and $c_{s}$ will be small in those regions. Large $c_{p}$ values correspond to planar diffusion, large $c_{s}$ values corresponds to isotropic media.

Due to the structure of the heart muscle, we will use the sum of the $c_{\ell}$ and $c_{p}$ coefficients to characterize the tissue, thus looking for regions with high combined directional and planar anisotropies

$$
c_{\text {muscle }}=c_{\ell}+c_{p}=1-c_{s} .
$$

This approach retains all of the directional information from $c_{l}$ and $c_{p}$ and discards all nondirectional $c_{s}$. 


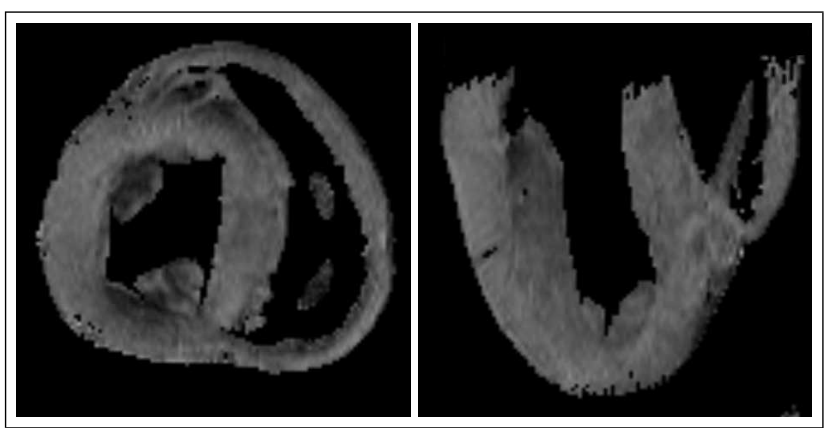

Figure 1: Grayscale mapped value of diffusion tensor anisotropy measure $c_{p}+c_{l}$ for the "short axes" and "long axes" views of the heart. The data is thresholded and all voxels with the signal strength below the threshold value are zero out.

\subsection{Algorithm}

We first use standard visualization techniques to investigate the range of parameters in the data and and locate the starting region for fiber tracing procedure. We then proceed with moving least squares (MLS) fiber tracing method developed in Zhukov and Barr [2002].

The basic steps of the MLS algorithm are:

1. Interpolate given $3 \mathrm{D}$ tensor data component-wise in the entire volume and create a continuous tensor field.

2. Choose the seed region for the fibers (can be the entire volume).

3. Trace fibers individually using Moving Least Square (MLS) method for dynamic regularization. Fibers will follow the regularized principal direction (direction of the eigenvector with largest eigenvalue) The tracing will stop when the fiber reaches the areas with below the threshold value of chosen anisotropy parameter.

4. Render individual fibers as streamlines or stream-tubes.

\subsection{Data}

The 3D DT-MRI data was collected from an intact fixed canine heart using reduced encoding and constrained reconstruction methodology [Hsu and Henriquez 2001] and interpolated via zero filling to $256 \times 256 \times 256$ voxels, with a voxel side of $\sim 0.4 \mathrm{~mm}$. The data was thresholded and all voxels with the signal strength below the threshold value were zero out. Figure Fig. (1) shows grayscale mapped value of anisotropy measure $c_{p}+c_{l}$ for the "short axes" and "long axes" views of the heart.

\subsection{Computations}

Tracing fibers requires computation of weighted averages of tensor fields within the filter radius (1-3 voxel sizes) and finding tensor eigenvalues and eigenvectors on every step. These are the most expensive computations in the algorithm and are performed using SVD decomposition and LU factorization using routines from [Press et al. 1992]. We employ standard Euler and Runge-Kutta forward integration scheme [Press et al. 1992] to evaluate the line integrals in tracing procedure.

\subsection{Visualization}

We use two major techniques to visualize tensor data - glyph based visualization and fiber tracing. We notice [Worth et al. 1998] that three-dimensional boxes used as glyphs in the complicated tensor fields convey information better than ellipsoids. We apply boxbased visualization to the short axis (axial) slice of the data. The results are shown in Fig. (2), where boxes are scaled according to $\left(\lambda_{1}, \lambda_{2}, \lambda_{3}\right)$ eigenvalues and oriented according to corresponding eigenvectors. As seen in Fig.(2), left and middle images, boxes have almost equal sides, i.e. in our data first eigenvalue is only slightly larger than the second and third (see Discussion Section). In order to visually enhance the directionality of the field we use $\left(\lambda_{1}-\lambda_{3}, \lambda_{2}-\lambda_{3}, \lambda_{3} / 10\right)$ for scaling factors in further visualization. (see Figs. (2-3)).

In the fiber visualization, fibers are grown along the "largest eigenvector" - the eigenvector corresponding to the largest eigenvalue of the regularized continuous tensor field. The integration step size is taken to be 0.1 of the data voxel size and the moving filter covers $1-3$ of neighboring voxels. The seeding regions for the fibers are chosen to be a thick (10 voxels) vertical slab going through the center of the dataset and with width and height covering the entire data volume. The seeding points are located on a regular grid $\{20 \times 20 \times 5\}$ within the slab, but the lines start only from the points with $c_{p}+c_{l}>\epsilon$, with $\epsilon=0.1$ for this dataset. For more details and explanation of the fiber tracing technique see Zhukov and Barr [2002].

We employ several distinct color-mapping schemes for visualization. We use direct RGB $\rightarrow$ XYZ mapping for all glyph based visualization and some of fiber tracking results. In this scheme, the elements oriented along the $\mathrm{X}$ axis are colored red, along the $\mathrm{Y}$ axis - green and Z axis - blue (see Figs. (2-4)).

For heart muscle visualization, it is important to be able to distinguish between clockwise and counter-clockwise orientation of spiraling fibers corresponding to left and right handed spirals. To emphasize that difference, we developed two color-mapping schemes. The first one classifies fibers according to the chirality (right or left handedness) without taking into account the value of the pitch. All fibers with the same chirality are colored the same way. Such colormapping is useful for classification purposes, but creates abrupt changes in color (singularities) on the boundaries (see Fig. (5)).

The second method adds the pitch angle to the scheme and allows the mapping to smoothly blend colors from the most saturated, corresponding to the pitch angle of 45 degrees, to neutral for horizontal and vertical fibers (see Fig. (6)).

Both color-mapping schemes rely on the same idea: after choosing an axis of rotation for the spiral one can determine its chirality by comparing the $\mathrm{z}$ component of the cross product between the radius vector and tangent vector to the fiber at the chosen point and the $\mathrm{z}$ component of the tangent vector itself. Mathematically, if $\mathbf{r}$ the radius vector pointing to the element and $\tau$ is a tangent vector at the point, then

$$
g=\tau_{z} \cdot(\mathbf{r} \times \tau)_{z}
$$

If $g>0$, it is a clockwise (right handed) spiral, otherwise it is counter-clockwise (left handed).

\subsection{Rendering}

Visualization software is written using OpenGL and GLUT. DTMRI anisotropy for the volume is shown using 2D texture mapping on the three orthogonal planes. Fibers are rendered using illuminated streamline techniques described in Banks [1994] and Hege [1996] with diffuse and specular terms. Stream tubes are rendered as a longs sequence of cylinder primitives with axes following the fiber.

For glyph based rendering, we choose to form rotation and scaling matrices from eigenvectors and eigenvalues for every voxel of the original data and then apply them to cubes positioned in the center of those voxels. 
The direction of eigenvectors and magnitude of corresponding eigenvalues together with anisotropy measures are pre-computed to allow interactive frame rate during visualization.

All renderings are performed using NVIDIA GeForce4 Ti4200 video card on Pentium4 $2.80 \mathrm{GHz}$ PC with $1 \mathrm{~GB}$ of RAM.

\section{Discussion}

The mammalian heart primarily consists of four chambers: two ventricles at the bottom of the heart, and two atria above [Streeter 1979]. The heart also contains other structures, such as the valves between the chambers and the papillary muscles controlling the valves.

The heart pumps the blood along a multi-part pathway between the atria and ventricles. Since a muscle fiber can contract only in one direction, the heart structure is complex, to succeed at pumping the blood. Anatomically, to achieve this, the muscle walls of the ventricles and atria are composed of a single helically folded muscular structure, whose fibers continuously connect from one part of the heart to another [Streeter et al. 1978]. A contraction wave passes through this muscular helix, which creates the complex pumping cycle of the heart. The details in the geometric aspects of the helical structure determines the proper functioning of the heart. It is proposed that a better understanding of this helical structure can be used to more effectively correct impaired heart function with surgery [Buckberg et al. 2001].

Physically, muscle consists of many honeycombed "sarcomeres," consisting of interdigitating myosin filaments, in an actin matrix. Water molecules have difficulty in crossing cell membranes and the sarcomere boundaries. It is much easier for water molecules to diffuse to different parts within the same cell and within the same sarcomere than it is for them to cross into other cells or into other sarcomeres. Thus, in muscle tissue, water molecules diffuse in a preferential direction - they are more free to move up and down the length of the muscle fiber.

Due to these diffusion properties, muscle tissue can be characterized by one somewhat larger eigenvalue of the diffusion tensor, whose eigenvector is associated with the axial direction of the fiber, and two somewhat smaller eigenvalues; the eigenvectors for these should be perpendicular to the direction of the muscle fiber. Since there is a great deal of structure within the sarcomere, the large eigenvalue and the smaller eigenvalues are not vastly different from one another.

Our main results are presented in Figs. (4-6). Heart muscle fibers in Fig. (4) are colored according to RGB-XYZ color scheme. The spiraling diagonal orientation of the muscle fibers on the inside and outside surfaces of the heart reaches $\sim 60$ degrees angle with respect to the vertical. In Figs. (5- 6) the color scheme is sensitive to clock/counterclockwise direction of the spiraling muscle fibers. Clockwise spiral orientation on the inside surface of the heart (endocardium) shown in purple, and counterclockwise muscle fiber orientation on the outside surface of the heart (epicardium) is shown in blue.

\section{Conclusions}

In this paper we presented the results of visualization of a DT-MRI dataset of a canine heart using several visualization techniques: scalar field rendering, tensor glyph and fiber reconstruction methods.

Our results clearly show the 3D spiraling diagonal orientation of the muscle fibers on the heart and systematic smooth variation in pitch and direction of the fibers from endocardium to epicardium.

\section{Acknowledgments}

We would like to thank Prof. Edward W. Hsu, Department of Bioengineering, Duke University, for providing the dataset, technical discussions and valuable comments on the paper; Dr. John Wood for medical and anatomical consultations and visualization suggestions and Prof. David Banks for suggestions on rendering.

\section{References}

BANKS, D. C. 1994. Illumination in diverse codimensions. In Proceedings of the 21st annual conference on Computer graphics and interactive techniques, ACM Press, 327-334.

Basser, P., AND Pierpaoli, C. 1996. Microstructural and physiological features of tissues elucidated by quantitative-diffusion-tensor mri. $J$. Magn. Res. Ser. B 111, 209-219.

Basser, P., Mattiello, J., And LeBihan, D. 1994. Mr diffusion tensor spectroscopy and imaging. Biophysical Journal 66, 259-267.

Basser, P., Pajevic, S., Peropaoli, C., Duda, J., And Aldroubi, A. 2000. In vivo fiber tractography using dt-mri data. Magnetic Resonance in Medicine 44, 625-632.

Buckberg, G., Coghlan, H., and Torrent-Guasp, F. 2001. The structure and function of the helical heart and its buttress wrapping. Semin Thorac Cardiovasc Surgery 13, 358-385.

Hsu, E., AND HenRiQuez, C. 2001. Myocardial fiber orientation mapping using reduced encoding diffusion tensor imaging. Journal of Cardiovascular Magnetic resonance 3, 339-347.

PArker, G., Wheeler-Kingshott, C., And BArker, G. 2001. Distributed anatomical brain connectivity derived from diffusion tensor imaging. In IPMI'2001, XVIth International Conference on Information Processing in Medical Imaging, in Lecture Notes in Computer Science, vol. 2082, 106-120.

Poupon, C., Mangin, J.-F., Clark, C., V.Frouin, J.Regis, Bihan, D., AND BLOCH, I. 2001. Towards inference of human brain connectivity form mr diffusion tensor data. Medical Image Analysis 5, 1-15.

Press, W., Teukolsky, S., Vetterling, W., and Flannery, B. 1992. Numerical Recipes in C. Cambridge University Press.

Sachse, F. B., Henriquez, C., Seemann, G., Riedel, C., Werner, C. D., Penland, R. C., Davis, B., , And Hsu., E. 2001. Modeling of fiber orientation in the ventricular myocardium with $\mathrm{mr}$ diffusion imaging. In Proc. Computers in Cardiology, vol. 28, 617-620.

Streeter, D., Powers, W. E., Ross, A., And Torrent-GuAsp, F. 1978. Cardiovascular System Dynamics. Cambridge: M.I.T. Press, ch. Three-Dimensional Fiber Orientation in the Mammalian Left Ventricular Wall, 73.

Streeter, D. 1979. The Cardiovsacular System. Baltimore: Williams and Wilkins, 61-112.

Tseng, W.-Y. I., Reese, T. G., Weisskoff, R. M., And WedeEn, V. J. 1999. Cardiac diffusion tensor mri in vivo without strain correction. Magnetic Resonance in Medicine 42, 2, 393-403.

Westin, C.-F., Peled, S., Gubjartsson, H., Kikinis, R., Jolesz, F., AND KIKINIS, R. 1997. Image processing for diffusion tensor magnetic resonance imaging. In Proceedings of ISMRM'97.

Worth, A. J., Makris, N., Wedeen, V. J., CaViness, J. V. S., AND KENNEDY, D. N. 1998. Fusion of mri data for visualization of white matter bundles. Tech. rep., http://neurowww.mgh.harvard.edu/cma/staff/ajw/MICCAI98/MICCAI98.html.

ZCKler, M., Stalling, D., AND Hege, H.-C. 1996. Interactive visualization of $3 \mathrm{~d}$-vector fields using illuminated stream lines. In Proceedings of the conference on Visualization '96, IEEE Computer Society Press, 107-ff.

ZHUKOV, L., AND BARR, A. H. . 2002. Oriented tensor reconstruction: Tracing neural pathways from diffusion tensor mri. In IEEE Visualization 2002 Proceedings, 387-394. 

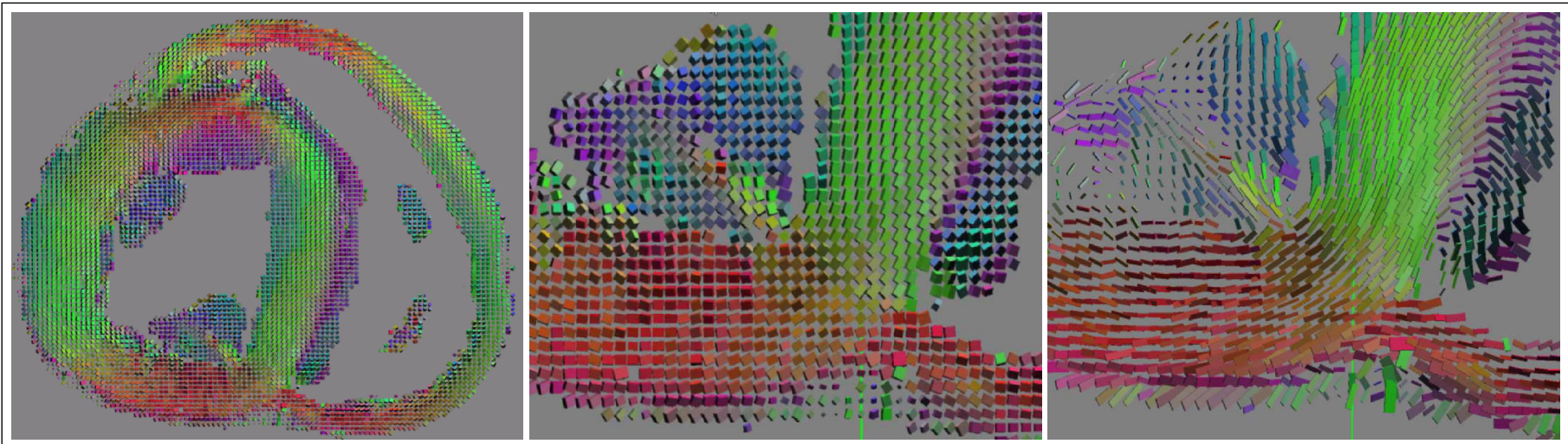

Figure 2: Glyph-based visualization of the the raw data tensors. RGB colors correspond to XYZ components of the largest eigenvector. Boxes are oriented according to eigenvectors and scaled by eigenvalues. For example, red boxes are oriented along the red (X) axis. Blue boxes correspond to the vertical muscle fibers in the inside the ventricle. From left to right: axial slice of the data; magnified region from the left image; the same region with enhanced scaling for the glyph boxes emphasizing principal direction. Note the transition from the red boxes along the $\mathrm{X}$ axis to the green boxes, parallel to $\mathrm{Y}$ axis. This transition follows the direction of the heart muscle fibers.

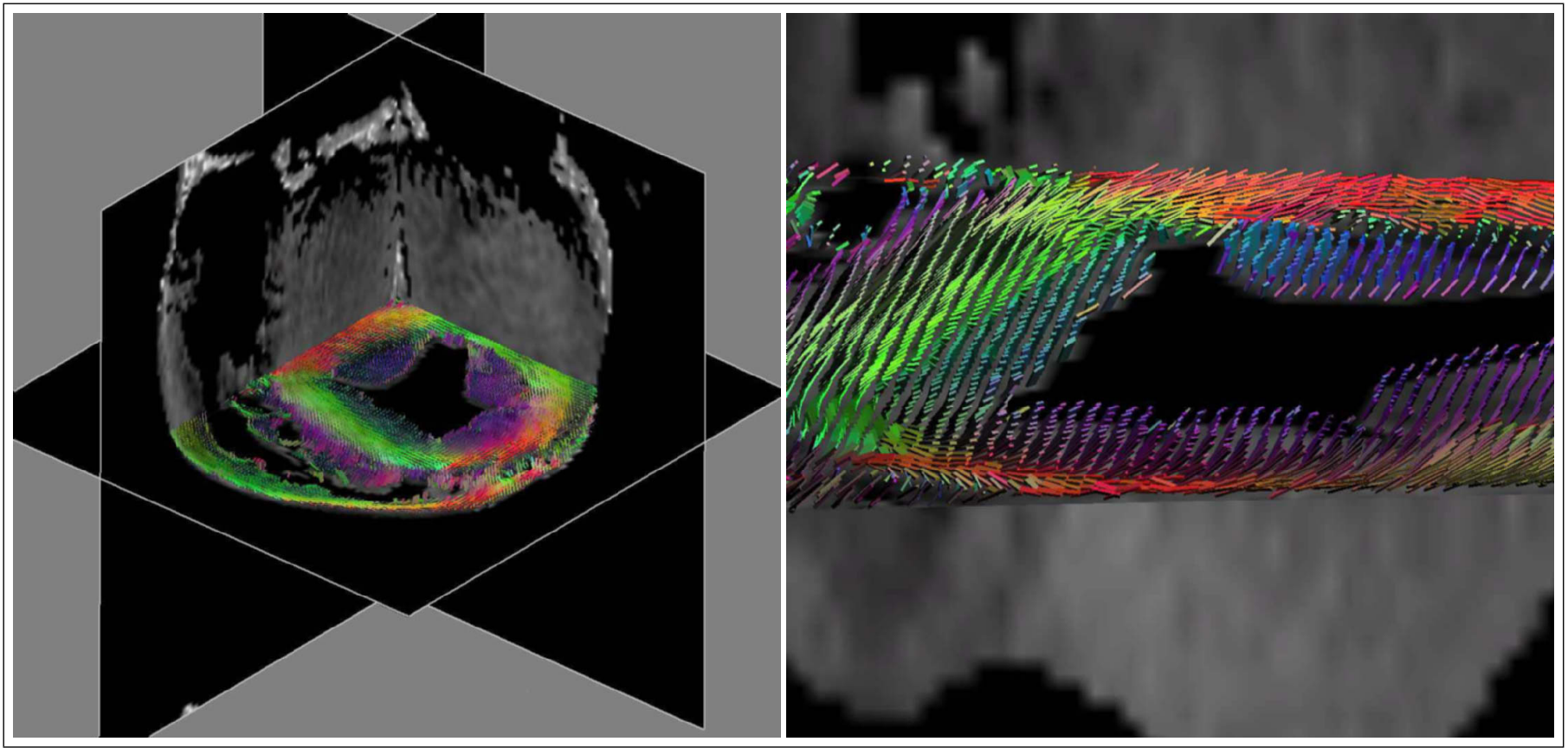

Figure 3: Glyph based visualization of diffusion tensor shown on the background of anisotropy measure: left - the entire dataset; right image - zoomed in view. Notice the vertical (blue) component of the boxes and the "flatter" orientation of the red boxes. 


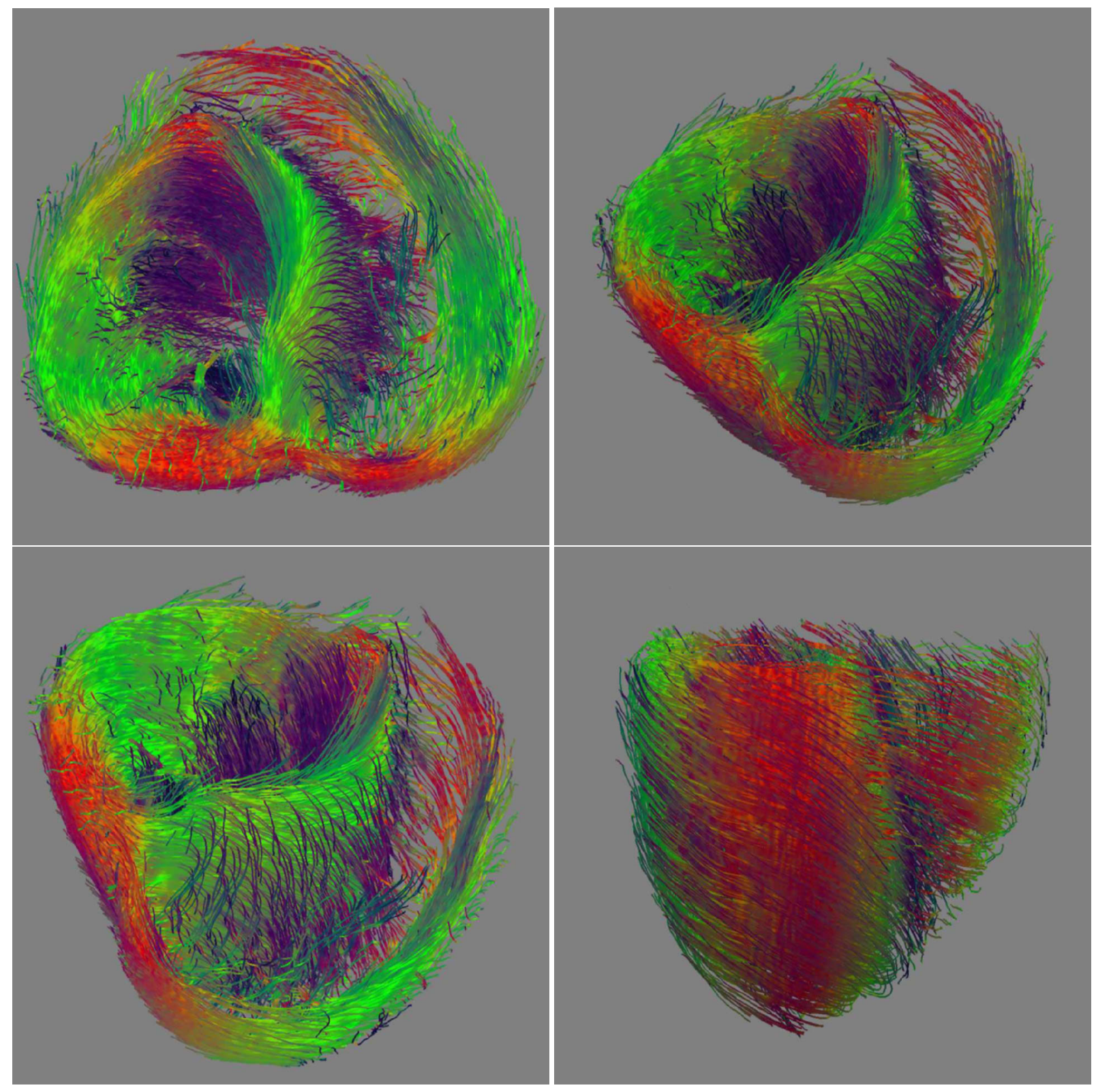

Figure 4: Reconstruction of heart muscle fibers using MLS algorithm. This image uses the RGB-XYZ color scheme. Notice spiraling diagonal orientation of the muscle fibers on the inside and outside surfaces of the heart. 




Figure 5: In this figure we use a color scheme which is sensitive to clock/counterclockwise direction of spiraling muscle fibers. Clockwise spiral orientation on the inside surface of the heart (endocardium) shown in purple, and counterclockwise muscle fiber orientation on the outside surface of the heart (epicardium) is shown in blue. This result illustrates the hypothesis that the spiraling fiber orientation in the heart muscle changes from endocardium to epicardium. The fibers are color-mapped independently on how steep the pitch is, causing abrupt change of color on the top.

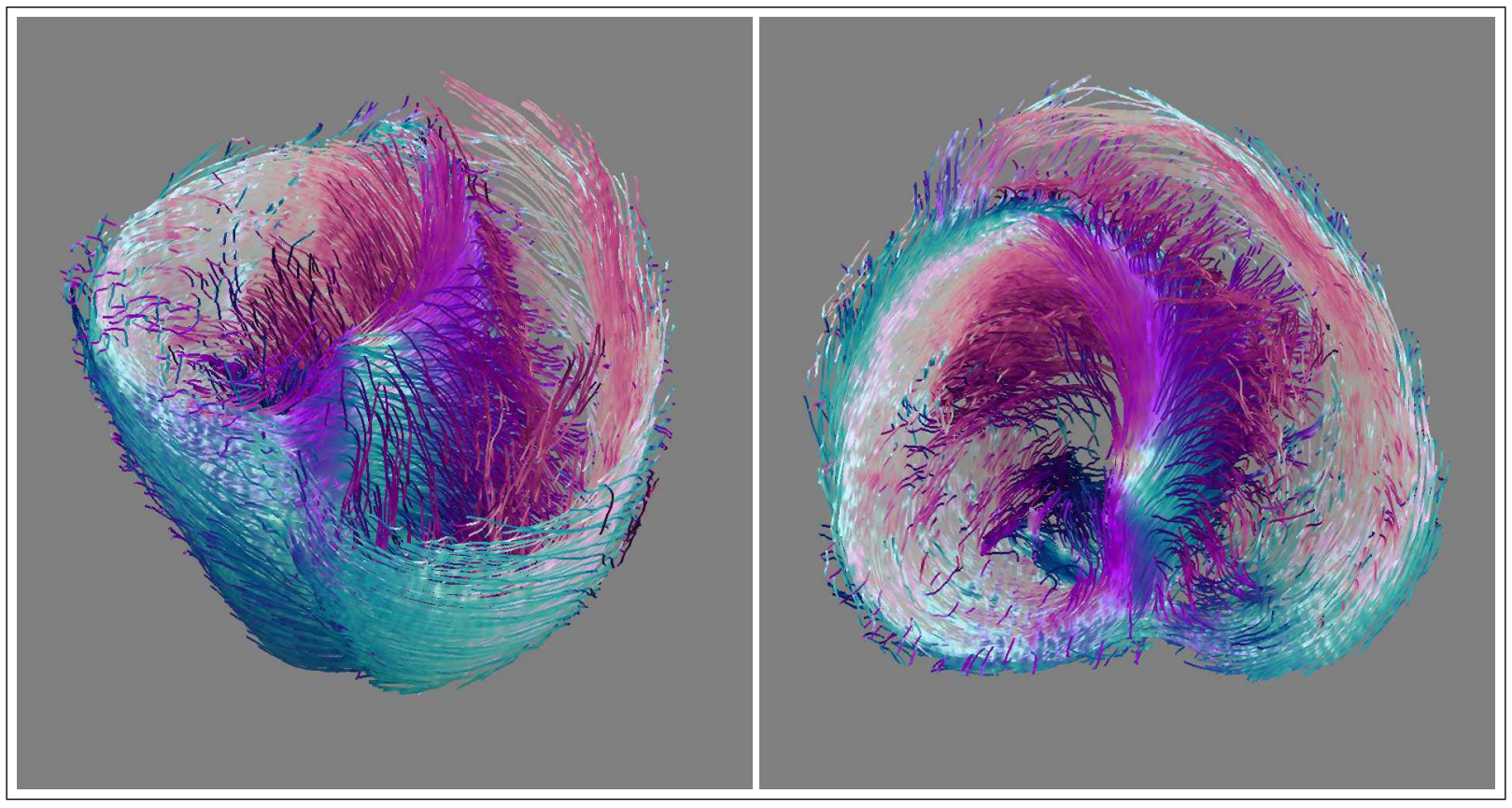

Figure 6: The color scheme used in this figure changes smoothly from clockwise to counterclockwise spiral oriented fibers. Horizontal parts (very small pitch angle) of the fibers are shown in white. This coloration is consistent with observations of some heart researchers, who have described a systematic smooth variation in pitch and direction of heart muscle fibers from endocardium to epicardium. 